\title{
Editorial
}

\section{French-German Texture and Anisotropy Meeting}

\author{
Claude Esling, ${ }^{1}$ Günter Gottstein, ${ }^{2}$ Richard Penelle, ${ }^{3}$ and Werner Skrotzki ${ }^{4}$ \\ ${ }^{1}$ Laboratoire d'Etude des Textures et Application aux Matériaux (LETAM), CNRS UMR 7078, University of Metz, \\ Ile du Saulcy, 57045 Metz, France \\ ${ }^{2}$ Institut für Metallkunde und Metallphysik, RWTH Aachen University, 52056 Aachen, Germany \\ ${ }^{3}$ Laboratoire de Physico-Chimie de l'Etat Solide, UMR 8182, Bâtiment 410, Universté Paris-Sud XI, \\ 15 rue Georges Clémenceau, 91405 Orsay Cedex, France \\ ${ }^{4}$ Institut für Strukturphysik, Technische Universität Dresden, 01062 Dresden, Germany
}

Correspondence should be addressed to Werner Skrotzki, werner.skrotzki@physik.tu-dresden.de

Received 25 May 2008; Accepted 25 May 2008

Copyright (c) 2008 Claude Esling et al. This is an open access article distributed under the Creative Commons Attribution License, which permits unrestricted use, distribution, and reproduction in any medium, provided the original work is properly cited.

The main topic of the meeting in Aachen of the working groups "Textures and Anisotropy" of the French and German Metallurgy and Materials Societies was "Texture and processing." Indeed, the crystallographic texture and microstructure, which closely depend on the processing route and chemistry of materials, determine their physical and mechanical properties.

At the macroscopic scale, texture and microstructure until the final product are tightly linked to the successive steps of the processing route, that is, solidification, hot working (forging, rolling, etc.), classical cold working or severe deformation, heat treatments, recrystallization annealing. In fact, texture and microstructure comprise grain size and shape, the spatial distribution of the actual phases, the spatial distribution of grain orientations which is described by the orientation density function, the spatial distribution of grain misorientations which is associated with the nature of grain boundaries.

At the microscopic scale, the main physical mechanisms occurring throughout the processing route are the following.

(i) The first mechanism is crystallization from a noncrystalline state generally starting from the liquid state: during this solidification step, large columnar grains, with a $\langle 100\rangle$ direction for cubic materials, develop perpendicular to the ingot or slab surface, except when a strong electromagnetic mixing is operating. All the final microstructures and textures will obviously be affected by this first solidification stage; however, phase transformations can dramatically change the textures by randomization or by creating a new texture (transformation texture) according to Burgers relations, for instance. (ii) The second mechanism is precipitation and/or phase transformations by diffusion and/or martensitic transformation during hot working or heat treatments.

(iii) The third mechanism is slip and dynamic recrystallization during hot working, slip, and/or twinning during cold deformation.

(iv) The fourth mechanism is primary recrystallization during annealing of the cold worked state as well as normal and abnormal grain growth.

(v) The fifth mechanism is rigid body rotation of particles, due to mechanical, electrical, or magnetic forces.

The studies of crystallographic texture primarily deal with metals, including intermetallics, but also ceramics, geological materials, and some organic materials such as polymers. The crystallographic texture and the related grain boundary distribution affect the intragranular and intergranular properties, respectively.

At the intragranular scale, the presence of preferred orientations is at the origin of the anisotropic behavior of structural materials such as earing during deep drawing of cans or ridging during sheet forming of ferritic steels. This plastic anisotropy can be described by various anisotropy coefficients. In order to control plastic anisotropy, online texture determination equipment is in use, with a low resolution which is, however, sufficient to give a reasonable approximation of the low-order anisotropy coefficients.

The presence of texture can also play an important role for in-service properties of functional materials. Remarkable examples are cladding tubes for nuclear fuel, high temperature superconductors, ceramic substrates, magnetic properties of thin films, or of Fe-3\%Si electrical steels used 
in transformer cores. However, the behavior of textured materials can be inhomogeneous if the spatial distribution of grain orientations is not random. For instance, the presence of grain clusters of similar orientation which can be compared to coarse grains or a single crystal embedded in a fine grain matrix is totally unacceptable for the fatigue resistance or toughness.

At the intergranular scale, the spatial distribution of the nature of grain boundaries affects important properties such as stress corrosion resistance, especially if percolation of a given grain boundary type sensitive to embrittlement is observed. Moreover, grain boundaries obviously play a major role in primary recrystallization and grain growth.

In conclusion, it is evident that the control of texture and microstructure represents a scientific and industrial challenge of prime importance to predict and optimize material properties, challenge on which the German and French "Texture and Anisotropy" groups are working.

Claude Esling Richard Penelle Günter Gottstein Werner Skrotzki 